\title{
Singular Two-Point Boundary Value Problems of Finite Element method
}

\author{
Weiwei Zhang \\ University of Sciences, Hebei University of Technology, Beichen district, Tianjin 300401, China
}

\begin{abstract}
In this paper, a class of singular two-point boundary value problems using the Galerkin method, using hierarchical grid method in discrete space, using one yuan for discrete function respectively, is obtained the general grid on the norm of the error convergence.
\end{abstract}

Keywords: singular, two-point boundary value problems, Galerkin method, super convergence, Finite Element method

\section{Introduction}

In this paper a yuan in hierarchical grid numerical solving the following singular two-point boundary value problems.

$$
\left\{\begin{array}{c}
-u^{\prime \prime}(x)-\frac{b}{x} u^{\prime}(x)+q(x) u(x)=f(x), \quad 0<x<1 \\
u^{\prime}(0)=0, \quad u(1)=0
\end{array}\right.
$$

Here is the constant, $b>0$ is responsible $q(x)$ for the function, $f(x)$ for a given function. [1], [2], [3] know if there is the only solution of this problem and smooth.

The singular boundary value problems can be caused by high dimensional non simplified spherically symmetric problems, the first side value problem of three dimensional elliptic equations:

$$
\left\{\begin{array}{l}
-\nabla(a \nabla u)=f \quad \text { in } \Omega \\
\left.u\right|_{\partial \Omega}=0
\end{array}\right.
$$

Through coordinate transformation into this problem.

This equation numerical Xie Ben Wen from the following several aspects: one, layered grid; Second, the equation of variational form; Three, equations of discrete; Four, error estimate.

Duran [7]in 2006 for the first time put forward the hierarchical grid, the grid to adjacent cell changes slowly, without considering the change of the thickness of the grid. Chuan-miao Chen[13]on special grid discussed the convergence of the differential error norm and superconvergence. Zhang $\mathrm{xu},[1],[12]$ on the basis of chuan-miao Chen made a general grid model and the error of the maximum modulus difference of convergence.

In this paper, on the basis of zhang $\mathrm{xu}$, do the general layered grid on the grid on the norm of the error convergence and convergence.

\section{The Discrete}

By the Green points male, can get the variational equation $a(u, v)=\int_{\Omega} x^{b} f v d x \quad, \forall v \in H^{1}(\Omega)$

$$
\begin{aligned}
& a(u, v)=\int_{0}^{1} x^{b} u^{\prime}(x) v^{\prime}(x)+x^{b} u(x) v(x) d x \quad v \in H^{1} \\
& \langle f, v\rangle=\int_{0}^{1} x^{b} f v d x \quad v \in H^{1}
\end{aligned}
$$

By [5] the problem (1) and (2.3), and other equivalent. The interval are partitioned into coarse grid and fine grid:

$\Omega_{1}=\left[0, \frac{1}{n}\right], \Omega_{2}=\left[\frac{1}{n}, 1\right]$. The fine grid $\Omega_{2}$ for subdivision: average composition, $\frac{1}{n}=x_{1}<\cdots<x_{n}=1$.

The $\mathrm{i}$ remember as a unit $I_{i}=\left[x_{i-1}, x_{i}\right], h_{i}=\frac{1}{n}$. The same as $\Omega_{1}, 0=x_{00}<x_{01}<\cdots<x_{0 n}=\frac{1}{n}$. The 1 i remember as a unit $I_{1 i}=\left[x_{1 i-1}, x_{1 i}\right], h_{i}=\frac{1}{n^{2}}$.

Define the finite dimensional test space $V_{h}$ is piecewise polynomial in hierarchical grid space, $V_{h}=\left\{v \in H_{0}{ }^{1}(\Omega):\left.v\right|_{\Omega_{i}} \in P_{k-1}\left(I_{i}\right), 1 \leq i \leq n\right\}$

Here $P_{k-1}\left(I_{i}\right)$ is polynomial representative the $i$ remember as a unit $^{I}, V_{h} \subset H^{1}$.

The equation can be turned into

$$
\begin{gathered}
a\left(u_{h}, v\right)=\int_{0}^{1} x^{b} f v d x \quad v \in H_{0}^{1} \\
\sum_{i=1}^{n} a\left(u_{i}, v_{i}\right)=\int_{0}^{1} x^{b} f v_{i} d x, \quad i=1,2, \cdots, n \\
\text { Norm: }\|v\|_{m, p, \Omega}=\left\{\sum_{|\alpha| \leq m}\left\|D^{\alpha} v\right\|_{0, p, \Omega}^{p}\right\}^{1 / p}
\end{gathered}
$$

Semi-norm: $|v|_{m, p, \Omega}=\left\{\sum_{|\alpha|=m}\left\|D^{\alpha} v\right\|_{0, p, \Omega}^{p}\right\}^{1 / p}$ 
Weight norm: $\|v\|_{m, p, \Omega}=\left\{\sum_{|\alpha| \leq m} x^{\alpha}\left\|D^{\alpha} v\right\|_{0, p, \Omega}^{p}\right\}^{1 / p}$

Weight semi- norm: $|v|_{m, p, \Omega}=\left\{\sum_{|\alpha|=m} x^{\alpha}\left\|D^{\alpha} v\right\|_{0, p, \Omega}^{p}\right\}^{1 / p}$

\section{The Convergence of Solution}

\subsection{Preliminary Knowledge}

Lemma1 [5] We assumption $u(x)$ and $u_{h}(x)$ are the solution of vanational problem and the solution of the discrete problem. Here, $\exists C=$ const $>0$, can be used

$$
\left\|u-u_{h}\right\| \leq C \inf _{v_{h} \in V_{h}}\left\|u-v_{h}\right\|
$$

Lemma2[8],[9],[10] Construct the interpolation postprocessing operator $\prod_{2 h}^{k}$, here $u_{I} \in V_{h}$ is the finite element interpolation for $u, k$ is the number of interpolation polynomia,so

$$
\begin{gathered}
C=\text { const }>0, \\
\left\|\prod_{2 h}^{k} u-u\right\|_{l} \leq C h^{r+1-l}\|u\| \\
\left\|\prod_{2 h}^{k} v\right\|_{l} \leq C\|v\|_{l}, \quad \forall v \in V_{h} \\
\prod_{2 h}^{k} u_{I}=\prod_{2 h}^{k} u
\end{gathered}
$$

When $l=0,1,0 \leq r \leq k$.

Lemma3[6] Assumption $u(x)$ and $u_{I}(x)$ is the solution of variational problem and linear interpolation ,

$$
a\left(u-u_{I}, v\right) \leq O\left(h^{2}\right)|u|_{3}\|v\|_{1}, \quad v \in V_{h}
$$

$\forall p \geq 2$ 和 $b>0$.

\subsection{Error estimation for $u-u_{I}$}

Theorem 1 Assumption $u(x)$ and $u_{I}(x)$ is the solution of variational problem and linear interpolation ,

$\forall p \geq 2$ and $b>0$ proof: In order to discuss on local unit, we introduce the following auxiliary function

$$
\phi(x)= \begin{cases}\int_{x_{j}}^{x_{j+1}} v^{\prime}(t) d t & 0 \leq x \leq x_{j-1} \\ \int_{x_{j}}^{x} v^{\prime}(t) d t & x_{j-1} \leq x \leq x_{j} \\ 0 & x_{j} \leq x \leq 1\end{cases}
$$

The auxiliary function $\phi(x) \in V_{h}$,

Use the affine transformation $x=x_{i-1}+h_{i} \xi$,here $\xi \in[0,1]$

in $I_{i}$.order $v_{h}=\phi(x)$, then

$x^{b}=\left(x_{i-1}+h \xi_{i}\right)^{b}=\left(x_{i} \xi+(1-\xi) x_{i-1}\right)^{b} \geq x_{i}^{b} \xi^{b}$

SO

$$
\begin{aligned}
\int_{0}^{1} x^{b}\left(u-u_{I}\right)^{\prime} v_{h}^{\prime} & =\int_{0}^{1} x^{b}\left(u-u_{I}\right)^{\prime} \phi^{\prime} d x \\
& =\int_{x_{i}}^{x_{i+1}}\left(v^{\prime}(x)\right)^{2} d x \\
& \geq h_{i} x_{i}^{b} \int_{0}^{1} t^{b}\left(v^{\prime}(x)\right)^{2} d x \\
& \geq C h_{i} x_{i}^{b} \max \left|v^{\prime}\right|_{1}^{2}
\end{aligned}
$$

and because

$$
\begin{aligned}
& \int_{0}^{1} x^{b}\left(u-u_{I}\right)^{\prime} v^{\prime}(x) d x \\
& =\sum_{i=0}^{n} \int_{x_{i}}^{x_{i+1}} x^{b}\left(u-u_{I}\right)^{\prime} v^{\prime}(x) d x \\
& =\sum_{i=0}^{n}\left[\left.x^{b}\left(u-u_{I}\right) v(x)\right|_{x_{i}} ^{x_{i+1}}-\int_{x_{i}}^{x_{i+1}} b x^{b-1}\left(u-u_{I}\right)^{\prime} v^{\prime}(x) d x\right] \\
& =\sum_{i=0}^{n}-b x^{b-1} \int_{x_{i}}^{x_{i+1}}\left(u-u_{I}\right)^{\prime} v^{\prime}(x) d x \\
& \leq C x^{-1} \sum_{i=0}^{n} \int_{x_{i}}^{x_{i+1}}\left(u-u_{I}\right)^{\prime} v^{\prime}(x) d x \\
& \leq C x^{-1} O\left(h^{2}\right)\|u\|_{3}|v|_{1}
\end{aligned}
$$

From Lemma3 :

$$
\begin{gathered}
a\left(u-u_{I}, v\right)=\int_{0}^{1}\left[x^{b}\left(u-u_{I}\right)^{\prime} v^{\prime}(x)+x^{b}\left(u-u_{I}\right) v(x)\right] d x \quad v \in H^{1} \\
\int_{0}^{1} x^{b}\left(u-u_{I}\right)^{\prime} v^{\prime}(x) d x<\int_{0}^{1}\left[x^{b}\left(u-u_{I}\right)^{\prime} v^{\prime}(x)+x^{b}\left(u-u_{I}\right) v(x)\right] d x \quad v \in H^{1}
\end{gathered}
$$$$
a\left(u-u_{I}, v\right) \leq C O\left(h^{2}\right)\|u\|_{3}|v|_{1}
$$

3.3 Error estimation for $u_{h}-u_{I}$

so:

$$
\left|u-u_{I}\right|_{1} \leq C h^{2}\|u\|_{3} .
$$

Theorem2 Assumption $u_{h}(x)$ and $u_{I}(x)$ are discrete problems and the solution of a linear interpolation, $\forall p \geq 2$ and $b>0$, then

$$
\left|u_{h}-u_{I}\right|_{1} \leq C h^{2}\|u\|_{3}
$$




\section{International Journal of Science and Research (IJSR) \\ ISSN (Online): 2319-7064}

Index Copernicus Value (2013): 6.14 | Impact Factor (2015): 6.391

proof: Take coefficient of variable $x=x_{e}$, because (2.1),

$$
\begin{aligned}
& a(u, v)=\int_{0}^{1} x^{b} u^{\prime}(x) v^{\prime}(x)+x^{b} u(x) v(x) d x \quad v \in V_{h} \\
& a\left(u_{h}, v\right)=\int_{0}^{1} x^{b} u_{h}{ }^{\prime}(x) v^{\prime}(x)+x^{b} u_{h}(x) v(x) d x \quad v \in V_{h}
\end{aligned}
$$

Two type is presupposed regularity,

$$
a\left(u-u_{h}, v\right)=0, \quad \forall v \in V^{h}
$$

so

$$
\begin{aligned}
& a\left(u-u_{I}+u_{I}-u_{h}, v\right)=0, \quad \forall v \in V^{h} \\
& a\left(u_{h}-u_{I}, v\right)=a\left(u-u_{I}, v\right) \quad \forall v \in V_{h}
\end{aligned}
$$

Because theorem1 then

$$
a\left(u-u_{I}, v\right)=O\left(h^{2}\right)\|u\|_{3}|v|_{1}, \quad \forall v \in V_{h}
$$

order $v=u_{h}-u_{I} \in V_{h}$

$$
\begin{aligned}
& C\left\|u-u_{I}\right\|^{2} \\
& \leq a\left(c, u_{h}-u_{I}\right) \\
& \leq a\left(u-u_{I}, u_{h}-u_{I}\right) \\
& =O\left(h^{2}\right)\|u\|_{3}\left|u_{h}-u_{I}\right|_{1}, \quad \forall v \in V_{h}
\end{aligned}
$$

Then

$$
\left|u_{h}-u_{I}\right|_{1} \leq C h^{2}\|u\|_{3}
$$

\subsection{The postprocessing for the Finite Element Method}

Theorem3 Assumption $u_{h}$ 和 $u_{I}(x)$ the solution of discrete problem and the solution of the linear interpolation, and meet the superclose property theorem 2 , then

$$
\left\|\prod_{2 h}^{2} u_{h}-u\right\|_{1} \leq C h^{2}\|u\|_{3}
$$

proof: Through the variable type for $\prod_{2 h}^{2} u_{h}-u$

$$
\prod_{2 h}^{2} u_{h}-u=\left(\prod_{2 h}^{2} u_{h}-\prod_{2 h}^{2} u_{I}\right)+\left(\prod_{2 h}^{2} u_{I}-u\right)
$$

Fist

$$
\left\|\prod_{2 h}^{2} u_{h}-\prod_{2 h}^{2} u_{I}\right\|_{1} \leq\left\|\prod_{2 h}^{2}\left(u_{h}-u_{I}\right)\right\|_{1}
$$

From Lemma2, $\prod_{2 h}^{k} u_{I}=\prod_{2 h}^{k} u$ so

$$
\left\|\prod_{2 h}^{2} u_{h}-\prod_{2 h}^{2} u_{I}\right\|_{1} \leq\left\|\Pi_{2 h}^{2}\left(u_{h}-u_{I}\right)\right\|_{1} \leq C\left\|u_{h}-u_{I}\right\|
$$

From theorem 3

$\left\|\prod_{2 h}^{2} u_{h}-\prod_{2 h}^{2} u_{I}\right\|_{1} \leq\left\|\Pi_{2 h}^{2}\left(u_{h}-u_{I}\right)\right\|_{1} \leq C\left\|u_{h}-u_{I}\right\| \leq C h^{2}\|u\|_{3}$

Second,from Lemma2

$$
\begin{gathered}
\left\|\prod_{2 h}^{k} u-u\right\|_{l} \leq C h^{r+1-l}\|u\|, \\
\left\|\prod_{2 h}^{k} v\right\|_{l} \leq C\|v\|_{l}, \quad \forall v \in V_{h} \text {, then } \\
\left\|\prod_{2 h}^{2} u_{h}-u\right\|_{1} \leq\left\|\prod_{2 h}^{2} u-u\right\|_{1} \leq C h^{2}\|u\|_{3} .
\end{gathered}
$$

3.5 Error estimation for $u-u_{h}$
Theorem4 Assumption $u(x)$ and $u_{I}(x)$ is the solution of variational problem and the solution of discrete problem, $\forall p \geq 2$ and $b>0$,

$$
\left|u-u_{h}\right|_{1} \leq C h^{2}\|u\|_{3}
$$

proof: From theorem1,3

$$
\left|u-u_{I}\right| \leq O\left(h^{2}\right)\left|u_{h}\right|_{3}\|v\|_{1}, \quad v \in V_{h}
$$

From theorem 2 and the triangle inequality

$$
\begin{aligned}
& \left|u-u_{h}\right|_{1}=\left|u-u_{I}+u_{I}-u_{h}\right|_{1} \\
& \leq\left|u-u_{I}\right|_{1}+\left|u_{I}-u_{h}\right|_{1} \leq C\left(h^{2}\right)\|u\|_{3}
\end{aligned}
$$

\section{Conclusion}

Chuan-miao Chen and zhang xu, is presented in this paper on the basis of the numerical solution of one dimensional singular pertueb- ation problems do the global superconvergence analysis, and got the expected results.

\section{References}

[1] X Zhang, "A class of singular nonlinear two-point boundary value problems of error estimate of Galerkin solution," Journal of computational mathematics, XXIII (4), pp. 195-205, 2010. (journal style)

[2] D. Jespersen, "Ritz-Galerkin Methods for Singular Boundary Value prob lems," Journal of of Siam Journal on Numerical Analysis, pp. 813-834, 1978. (journal style)

[3] K. Erikssonl, V Thomée, "Galerkin methods for Singular Boundary Value Problems in One Space Dimension," Journal of Mathematics of Computation, pp. 345-367, 1984. (journal style)

[4] Chen, C. M, "Superconvergence of galerkin solutions for singular nonlinear two-point boundary value problems," Journal of Mathematica Numerica Sinica, pp. 113-123, 1985. (journal style)

[5] Wang, $\mathrm{Xu}, \mathrm{R}$. Caves, The mathematical basis of the finite element method, Science Press, 2004. (book style)

[6] lin, Yan, Efficient finite element structure and analysis, Efficient finite element structure and analysis, Hebei university press, 1996. (book style)

[7] R G Dur á n, A L Lombardi, "Finite element approximation of convection diffusion problems using graded meshes," Journal of Applied Numerical Mathematics, pp. 1314-1325, 2006. (journal style)

[8] Lin, "Global error expansion and superconvergence for higher order interpolation of finite elements," In Proceedings of Applied Numerical Mathematics, 1992. (journal style)

[9] Q Lin, “A rectangle test for galerkin approximation to nonlinear hyperbolic problems," Journal of the Shandong Normal University, 1997. (journal style)

[10] Q Lin, “ The finite element interpolated correction method for elliptic eigenvalue problems," Chinese J.numer.math.appl, pp. 78-93, 1992. (journal style)

[11] D Jespersen , "Ritz-Galerkin Methods for Singular Boundary Value Problems[J]. Siam Journal on 


\section{International Journal of Science and Research (IJSR) \\ ISSN (Online): 2319-7064}

Index Copernicus Value (2013): 6.14 | Impact Factor (2015): 6.391

Numerical Analysis," Journal of Siam Journal on Numerical Analysis, pp. 813-834, 1978. (journal style)

[12] X Zhang, "A class of singular two-point boundary value problems of finite element method," Journal of Higher school, pp. 193-205, 2013. (journal style)

[13] M C Chen, "TGalerkin solution of singular nonlinear two-point boundary value problem of super convergence," Journal of computational mathematics, pp. 113-123, 1985. (journal style)

Volume 5 Issue 8, August 2016

www.ijsr.net 